\title{
No Populations Left Behind: Vaccine Hesitancy and Equitable Diffusion of Effective COVID-19 Vaccines
}

\author{
Monica Webb Hooper, $\mathrm{PhD}^{\top}$ (1), Anna María Nápoles, PhD, MPH², and \\ Eliseo J. Pérez-Stable, $M D^{7}$
}

\begin{abstract}
'Office of the Director, National Institute on Minority Health and Health Disparities (NIMHD), National Institutes of Health (NIH), Bethesda, MD, USA; ${ }^{2}$ Office of the Scientific Director, National Institute on Minority Health and Health Disparities (NIMHD), National Institutes of Health (NIH), Bethesda, MD, USA.
\end{abstract}

Racial/ethnic minority communities are experiencing an undue burden from coronavirus disease 2019 (COVID-19), and the availability of Food and Drug Administration (FDA) authorized vaccines is critical for improving population health. National surveys assessing vaccination willingness and reports of vaccination administration by race/ethnicity indicate at least two areas that warrant attention: elevated vaccine hesitancy among African American and Latino adults, and the need to ensure equitable access to vaccination. COVID-19 vaccine hesitancy is not uniform within racial/ethnic minority populations; yet, given the disproportionate impact, understandable distrust, and widespread misinformation, there is an imperative to overcome challenges associated with vaccination willingness and uptake, as well as implementation and access. This Perspective discusses the complexity of drivers for each of these areas, which include individual, community, and structural factors. It also highlights two initiatives at the National Institutes of Health. One is focused on addressing misinformation and distrust through academic-community partnerships, and the other on community-engaged behavioral interventions to address the population-specific reasons for COVID-19 vaccine hesitancy, support informed decisionmaking, and promote equitable access among populations with health disparities. For the foreseeable future, proactive and persistent efforts around COVID-19 mitigation strategies, including vaccination, will remain of paramount importance for health equity.

KEY WORDS: Vaccine; vaccine hesitancy; COVID-19; Severe acute respiratory syndrome coronavirus 2 ; racial and ethnic disparities; African Americans; Latinos.

J Gen Intern Med 36(7):2130-3

DOI: $10.1007 / \mathrm{s} 11606-021-06698-5$

(C) This is a U.S. government work and not under copyright protection in the U.S.; foreign copyright protection may apply 2021

$\mathrm{T}$ he coronavirus disease 2019 (COVID-19) pandemic has exacerbated long-standing health disparities, as racial/ ethnic minority populations have an undue burden in rates of

Received January 27, 2021

Accepted March 3, 2021

Published online March 22, 2021 infection, hospitalizations, and mortality. ${ }^{1}$ In December 2020, two vaccines were granted Food and Drug Administration (FDA) emergency use authorization (EUA) and a third vaccine was recently granted EUA. If ongoing trials are successful in obtaining EUAs, additional vaccines will be available within months. While many individuals have been waiting with bated breath for the availability of a safe and effective vaccine as a primary mechanism to end the pandemic, there are at least two significant areas that warrant attention. First, not everyone shares this elation - and vaccine hesitancy or suboptimal public willingness to accept the vaccine(s) among underserved populations may limit uptake even with widespread availability. Second, ensuring equitable access to COVID-19 vaccines is needed to end the pandemic and to provide much needed relief, particularly among populations affected disproportionately.

\section{THE CHALLENGE OF VACCINE HESITANCY}

Vaccine hesitancy is a complex cognitive and behavioral construct that varies for specific vaccines, places, and times. ${ }^{2}$ It represents the mid-point of a continuum ranging from complete refusal to full acceptance, and is influenced by complacency, convenience, and confidence. The biggest concern is that without intervention, hesitancy may shift to complete refusal or remain as passive avoidance to seek out immunization. Despite remarkable reductions in morbidity and mortality from infectious diseases since the early 1900s, the growing number of people electing to forgo vaccination jeopardizes efforts to achieve and sustain herd immunity in childhood infections. ${ }^{3}$ Racial/ethnic differences in vaccine hesitancy and subsequent uptake disparities in COVID-19 vaccination are of special concern.

Available evidence on racial/ethnic differences in COVID19 vaccine hesitancy is currently limited but expanding. Survey data from US respondents at multiple cross-sectional time points in 2020 and early 2021 indicated that the likelihood of COVID-19 vaccine acceptance varied by race/ethnicity. Overall acceptance was lower between May (42\% would definitely accept the vaccine) and September (21\% would definitely accept), and was 8 percentage points higher in November 2020 (29\% would definitely accept) relative to September. ${ }^{4}$ 
These reports also revealed racial/ethnic differences among those who indicated that they would "definitely" or "probably" accept a COVID-19 vaccine during the November assessment, with $42 \%$ of African American respondents reporting definitive/probable acceptance, followed by $61 \%$ of White, $63 \%$ of Hispanic/Latino, and $83 \%$ of Asian American respondents. Findings from a Kaiser Family Foundation (KFF) survey in January 2021 found greater enthusiasm for COVID-19 vaccines, yet elevated reluctance was identified among African American and Latino adults compared with White respondents. ${ }^{5}$ We can anticipate that acceptance will continue to improve over time, and assiduous efforts to reduce and ultimately eliminate racial/ethnic differences in willingness to accept vaccination are needed.

Data on the prevalence of completion of some common vaccines indicate that racial/ethnic minority populations are less likely to receive influenza and pneumococcal vaccinations. ${ }^{6,7}$ Among older adults, seasonal influenza vaccine uptake is lower among African American/Black, Latino, and Asian American persons, as well as individuals of lower socioeconomic status (SES), compared with their White and higher SES counterparts. ${ }^{8}$ Others have found that Asian American and African American/Black pregnant women were less likely to receive a clinician's recommendation for the influenza vaccine, and that vaccination was lower among African American expectant mothers. ${ }^{9}$ Some progress in vaccine coverage by race/ ethnicity has been made, as shown by the estimated vaccination rates of children born in 2016 and 2017, for which there are minimal differences among White, African American/Black, and Latino pediatric populations for measles, mumps, rubella, and polio with rates of 90 to $93 \%{ }^{10}$ While these epidemiological surveys did not focus on vaccine hesitancy, they highlight variations in uptake across vaccine types.

Drivers for COVID-19 vaccine hesitancy are multifaceted and dynamic. Commonly reported reasons for low vaccine confidence relate to concerns about the speed of development, potential harm from vaccine ingredients, yet-unknown longer term health effects, and unanswered questions about whether the vaccines prevent asymptomatic infection and transmission. There are also unique health concerns among medically vulnerable groups, such immunocompromised patients or those receiving immunomodulating drugs, as well as those with a history of severe allergies, which may also be deterrents to accept the vaccine(s). Among many, the desire to "wait and see" stems largely from these concerns. Furthermore, among African American individuals, low COVID-19 vaccine confidence is compounded with negative healthcare experiences ${ }^{11}$ and historical trauma due to biomedical science abuses ${ }^{12}$ that have synergistically contributed to institutional and interpersonal distrust. Research on disease-behavior dynamics suggests that population structure and the clustering of social networks that share the same vaccine-related attitudes are mechanisms that may underlie the persistence of vaccine hesitancy among certain groups of people. ${ }^{13}$ Indeed, the identification of clustered subpopulations with high hesitancy could be an important strategy to improve the uptake of COVID-19 vaccination using targeted approaches. Research is needed to understand and address these and other empirical questions. Proactively addressing vaccine hesitancy is of high priority for reducing racial/ethnic disparities in physical and mental health and economic well-being, and preventing gaps in education among children due to distance learning and associated digital inequities.

\section{COUNTERING THE COVID-19 INFODEMIC}

The depth of vaccination fear and distrust is further exacerbated by the COVID-19 infodemic, ${ }^{14}$ or the rapid and widespread dissemination of both empirically supported and inaccurate messages about the pandemic and the vaccines, fueling public confusion and distress. Aggressive efforts to combat COVID19 misinformation and disinformation, through simple, consistent, repetitive, and effective counter-messaging, are needed to increase confidence in the vaccines and the use of behavioral mitigation strategies. COVID-19 has heightened the need for trust and trustworthiness in science and medicine as the foundation on which to engage underserved communities in the healthcare system and prevent delays in help-seeking. Working across all societal sectors, not just healthcare, and with disproportionately affected communities to develop responsive messaging and interventions will be critical to diffusion efforts. Such communications to inform the public about safety standards for vaccine development and population health benefits must be delivered by trusted messengers and allow opportunities for individual questions to be addressed meaningfully. Informing clinicians about targeted strategies for motivating uptake - especially focusing on the reasons for hesitancy — will also be imperative. These elements are among the foci of the National Institutes of Health (NIH) Community Engagement Alliance Against COVID-19 Disparities (CEAL). Specific emphases within CEAL are to facilitate inclusive participation in COVID-19 clinical research (e.g., vaccine and therapeutic trials) among racial/ethnic minority populations and address misinformation and distrust around the FDA-authorized vaccines to promote uptake. CEAL work groups are developing and disseminating community competent educational resources for multiple stakeholders, including health professionals, scientists, and community members (visit: covid19community.nih.gov). CEAL research teams are also engaging local leaders and conducting a range of activities at the grassroots level including hosting webinars, townhalls, and local community meetings, as well as text messaging campaigns, and disseminating accurate and culturally appropriate materials.

\section{EQUITABLE ACCESS TO VACCINES}

Beyond hesitancy as an individual-level factor, ensuring equitable access to the COVID-19 vaccine(s) must be a societal 
priority. Vaccine administration data by race/ethnicity are incomplete much like data from the pandemic cases, but the extant evidence points to racial/ethnic disparities in completion of both doses 1 and 2 (of the currently available 2-dose regimen). Specifically, the rates of vaccination among African American and Latino individuals are lower than what would be expected based on their population sizes and COVID-19 burden in defined communities. ${ }^{15}$ Importantly, these lower vaccination rates are not attributable fully to vaccine hesitancy. Structural factors such as limitations in the cold-supply chain of vaccines, inadequate access to vaccine distribution clinics in underserved geographic areas, the digital divide (i.e., access to the internet to schedule appointments) and digital inequalities (i.e., internet use skills), and competition for limited appointments are contributors. Moreover, the potential subsequent vaccine reactions that could require missing work, the need to return for a second dose, and the unique concerns of undocumented immigrant patients represent structural barriers. Additionally, there are specific populations with greater vulnerability that our health care system needs to target in order to vaccinate such as older adults living alone, isolated rural communities, and persons living with severe mental illness. Research is needed to identify and address these and other structural inequities.

For scientists at the National Institute on Minority Health and Health Disparities (NIMHD), efforts to recognize, understand, and address the population-specific reasons for COVID-19 vaccine hesitancy and to promote equitable access are important. Systematic data collection and accurate reporting on COVID-19 vaccine availability and uptake by race/ethnicity, SES, and other characteristics will be needed to assess population coverage and equity. Even with the availability of vaccines, disparities will persist and may widen if the underlying drivers of vaccine adoption and variation across population groups are not addressed. COVID-19 vaccine hesitancy is not unique to racial/ethnic minority populations and there is significant within-group heterogeneity that must be recognized. Yet, given the disproportionate COVID-19 impact, elevated distrust due to past and modern-day experiences, and the infodemic, there is an imperative to overcome the challenges associated with COVID-19 vaccine implementation and uptake.

\section{SCIENCE RESPONSE FROM NIMHD}

Many scientific questions can be asked to increase our understanding of COVID-19 vaccine adoption. NIMHD is leading an NIH-wide Notice of Special Interest (NOSI), Research to Address Vaccine Hesitancy, Uptake, and Implementation among Populations that Experience Health Disparities (NOT-MD-21-008). This opportunity will support studies to examine racial/ethnic differences in beliefs and concerns about the COVID-19 vaccine(s) as well as other vaccinations (e.g., pneumococcal, influenza, hepatitis $\mathrm{B}$, human papilloma virus
(HPV), and herpes zoster), but most needed are effective interventions to reduce misinformation, build community trust, and promote dissemination and population-level uptake. We recommend and will support research to identify multilevel drivers of COVID-19 vaccine adoption and diffusion into specific communities that experience health disparities. Potential interventions to evaluate might include community responsive vaccine-related educational interventions, disinformation counter-messaging, implementation strategies in clinical and community settings, and effective health care system programs to vaccinate the most vulnerable. Other examples include policy, community-level, health system, interpersonal/ family/professional, and individual-level interventions that maximize vaccine access, uptake, and series completion. The goal is to maintain high rates of vaccination to prevent future outbreaks of this preventable disease. Controlling COVID-19 by equitable diffusion of the vaccines, continued and intensified testing, accurate messages about risks and benefits, and sustainment of mitigation behaviors will be the most important preventive strategies for promoting health equity in 2021 and beyond.

Corresponding Author: Monica Webb Hooper, PhD; Office of the Director, National Institute on Minority Health and Health Disparities (NIMHD), National Institutes of Health (NIH), Bethesda, MD, USA (e-mail: monica.hooper@nih.gov).

\section{Declarations:}

Conflict of Interest: The authors declare that they do not have a conflict of interest.

\section{REFERENCES}

1. Rossen LM, Branum AM, Ahmad FB, Sutton P, Anderson RN. Excess deaths associated with COVID-19, by age and race and ethnicity - United States, January 26-October 3, 2020. MMWR Morb Mortal Wkly Rep. 2020;69(42):1522-1527.

2. World Health Organization. Report of the SAGE Working Group on Vaccine Hesitancy 2014. Accessed December 21, 2020.

3. Siddiqui M, Salmon DA, Omer SB. Epidemiology of vaccine hesitancy in the United States. Hum Vaccin Immunother. 2013;9(12):2643-2648.

4. Pew Research Center. Intent to Get a COVID-19 Vaccine Rises to $60 \%$ as Confidence in Research and Development Process Increases. 2020. https://www.pewresearch.org/science/2020/12/03/intent-to-get-acovid-19-vaccine-rises-to-60-as-confidence-in-research-and-development-process-increases/. Accessed December 20, 2020.

5. Hamel L, Kirzinger A, Lopes L, Kearney A, Sparks G, Brodie M. $K F F$ COVID-19 Vaccine Monitor: January 2021. San Francisco, CA: Kaiser Family Foundation; February 25, 20212021.

6. Centers for Disease Control and Prevention. Flu Vaccination Coverage, United States, 2019-20 Influenza Season. National Center for Immunization and Respiratory Diseases (NCIRD). https://www.cdc.gov/flu/ fluvaxview/coverage-1920estimates.htm\#table1. Published 2020. Accessed December 21, 2020.

7. Centers for Disease Control and Prevention. Vaccination Coverage among Adults in the United States, National Health Interview Survey, 2017. National Center for Immunization and Respiratory Diseases. https:// www.cdc.gov/vaccines/imz-managers/coverage/adultvaxview/pubs-resources/NHIS-2017.html. Published 2017. Updated February 2, 2018. Accessed December 21, 2020.

8. Okoli GN, Abou-Setta AM, Neilson CJ, Chit A, Thommes E, Mahmud SM. Determinants of Seasonal Influenza Vaccine Uptake Among the 
Elderly in the United States: a Systematic Review and Meta-analysis. Gerontology and Geriatric Medicine. 2019;5:2333721419870345.

9. Arnold LD, Luong L, Rebmann T, Chang JJ. Racial disparities in U.S maternal influenza vaccine uptake: results from analysis of Pregnancy Risk Assessment Monitoring System (PRAMS) data, 2012-2015. Vaccine. 2019;37(18):2520-2526.

10. Hill HA, Yankey D, Elam-Evans LD, Singleton JA, Pingali SC, Santibanez TA. Vaccination coverage by age 24 months among children born in 2016 and 2017 - National Immunization Survey-Child, United States, 2017-2019. MMWR Morb Mortal Wkly Rep. 2020;69(42):15051511.

11. Hamel L, Lopes L, Munana C, Artiga S, Brodie M. Race, health, and COVID-19: the views and experiences of Black Americans. San Francisco, CA: Kaiser Family Foundation; October 13, 20202020.

12. Webb Hooper M, Mitchell C, Marshall VJ, et al. Understanding multilevel factors related to urban community trust in healthcare and research. Int $J$ Environ Res Public Health. 2019; 16(18).
13. Wang Z, Andrews MA, Wu ZX, Wang L, Bauch CT. Coupled diseasebehavior dynamics on complex networks: a review. Phys Life Rev. 2015;15:1-29.

14. Joint statement by WHO U, UNICEF, UNDP, UNESCO, UNAIDS, ITU, UN Global Pulse, and IFRC. Managing the COVID-19 infodemic: promoting healthy behaviours and mitigating the harm from misinformation and disinformation. World Health Organization. https://www.who.int/news/ item/23-09-2020-managing-the-covid-19-infodemic-promoting-healthybehaviours-and-mitigating-the-harm-from-misinformation-and-disinformation. Published 2020. Accessed December 21, 2020.

15. Centers for Disease Control and Prevention. Demographic characteristics of people receiving COVID-19 vaccinations in the United States. U.S. Department of Health and Human Services. COVID Data Tracker Web site. https://covid.cdc.gov/covid-data-tracker/\#vaccination-demographic. Published 2021. Updated February 24, 2021. Accessed February 24, 2021.

Publisher's Note: Springer Nature remains neutral with regard to jurisdictional claims in published maps and institutional affiliations. 